\title{
Long-term observation of magnetic pulsations through the ELF Hylaty station located in the Bieszczady Mountains (south-eastern Poland)
}

\author{
Zenon Nieckarz ${ }^{1, *}$ and Grzegorz Michałek ${ }^{2}$ \\ ${ }^{1}$ Marian Smoluchowski Institute of Physics, Jagiellonian University, ul. prof. St. Łojasiewicza 11, 30-348 Kraków, Poland \\ 2 Astronomical Observatory, Jagiellonian University, ul. Orla 171, 30-244 Kraków, Poland
}

Received 2 April 2020 / Accepted 22 October 2020

\begin{abstract}
Ground-based measurements of ultra- and extremely low-frequency waves (ULF/ELF) carried out in 2005-2016 (the 23rd and 24th solar cycle) at the ELF Hylaty station in Bieszczady Mountains (south-eastern Poland) were used to identify the days (360 days) in which magnetic pulsation events (MPEs) occurred. To reveal sources of MPEs at the Sun we considered their correlation with the basic indices describing solar activity. Our analysis, like earlier studies, did not reveal a significant positive correlation between the MPE detection rate and the sunspot numbers (SSN). On the other hand, we showed that MPEs are strongly correlated (correlation coefficient $\approx 0.70)$ with moderate $(D s t<-70 \mathrm{nT})$ and intense $(D s t<-100 \mathrm{nT})$ geomagnetic disturbances expressed by the Disturbance Storm Index (Dst). We recognized all sources of these geomagnetic storms associated with the considered MPEs. Only 44\% of the MPEs were associated with storms caused by CMEs listed in the CDAW LASCO CME catalog. 56\% of the MPEs were associated with storms caused by other phenomena including corotating interaction regions (CIRs), slow solar wind or CMEs not detected by LASCO. We also demonstrated that the CMEs associated with the MPEs were very energetic, i.e. they were extremely wide (partial and halo events) and fast (with the average speed above $1100 \mathrm{~km} \mathrm{~s}^{-1}$ ). CMEs and CIRs generally appear in different phases of solar cycles. Because MPEs are strongly related to both of these phenomena they cannot be associated with any phase of a solar cycle or with any indicator characterizing a 11-year solar activity. We also suggested that the low number of MPEs associated with CMEs is due to the anomalous 24 solar cycle. During this cycle, due to low density of the interplanetary medium, CMEs could easily eject and expand, but they were not geoeffective.
\end{abstract}

Keywords: magnetic pulsation / ULF/ELF waves / 24 Solar Cycle

\section{Introduction}

Earth's vicinity is mostly controlled by solar activity, especially by energetic events such as Coronal Mass Ejections (CMEs) and solar flares. Both of these phenomena significantly affect space weather: solar flares can cause a sudden change in the ionization level in the ionosphere, while CMEs are responsible for solar energetic particle (SEP) events and geomagnetic storms (Gopalswamy et al., 2001a; Gopalswamy, 2002; Zhang et al., 2003; Kim et al., 2005; Moon et al., 2005; Manoharan et al., 2004; Manoharan \& Agalya, 2011; Shanmugaraju et al., 2015; Klein \& Dalla, 2017). The human society has become increasingly dependant on technology placed in the space that can be easily affected by solar energetic events.

\footnotetext{
*Corresponding author: zenon. nieckarz@uj. edu.pl
}

Therefore, forecasting space weather is now an important issue (e.g. Schrijver, 2015; Knipp et al., 2018).

CMEs are believed to be the primary cause of the largest and most damaging geomagnetic storms. However, a geomagnetic storm is not the only effect related to the impact of CMEs on Earth. The discussion on the other space weather effects caused by CMEs continues in the next paragraphs as well. Extremely energetic CMEs associated with flares with unusually high intensity are considered the most extreme events at the Sun. The high magnetic fields carried by CMEs and high dynamic pressures associated with CME-driven shocks and sheaths can disturb the magnetic field of Earth generating severe geomagnetic storms (e.g. Burton et al., 1975; Farrugia et al., 1993; Cane et al., 2000; Kilpua et al., 2017). Fast CMEs additionally drive strong shocks that accelerate coronal particles up to $\mathrm{GeV}$ energies. These particles, along with extremely 
intense high-energy radiation from flares penetrate Earth's atmosphere causing a significant depletion of stratospheric ozone and an increase in the total content of electrons in the ionosphere. Therefore, predicting the occurrence of these phenomena is an important challenge for scientists. This is not a simple task because the expansion of CMEs depends on the magnetic force that drives them (physical conditions at the Sun) and the conditions prevailing in the interplanetary medium as well. In the initial phase, the magnetic force dominates and the ejection is accelerated rapidly. Farther from the Sun, the propelling force weakens and friction begins to prevail. Then the velocity of CMEs decreases slowly towards the speed of solar wind (e.g. Vršnak et al., 2004a, b; Zhang et al., 2001). In addition, the speed of ejections can change sharply as a result of CME-CME interactions (Gopalswamy et al., 2001b; Scolini et al., 2020; Lugaz et al., 2017). Such collisions mostly occur during solar activity maxima.

We must also mention that some major storms (disturbance storm time (Dst) index $\leq-100 \mathrm{nT})$ may be also driven by co-rotating interaction regions (CIRs) (Zhang et al., 2003, 2007; Richardson et al., 2006; Kilpua et al., 2017). CIRs are compression regions ahead of coronal holes high-speed stream (CH HSS). They form due to the interaction of slower, ambient solar wind ahead of the higher-speed stream originating from the more persistent coronal holes. CIRs are mostly known to be the primary cause of moderate $(-50 \mathrm{nT}>D s t>-100 \mathrm{nT})$ recurrent geomagnetic storms (particularly during solar minima (e.g., Verbanac et al., 2011; Echer et al., 2013).

As we mentioned earlier, CME reaching the Earth not only can generate geomagnetic storms, but can also trigger many phenomena detected in the Earth's magnetosphere. One of these are electromagnetic ion-cyclotron (EMIC) waves. These waves are generated by the proton cyclotron instabilities when energy transfer occurs between different particle populations via waveparticle interactions (Kitamura et al., 2018). EMIC waves can propagate along magnetic field lines to the Earth's surface and can be recorded by ground magnetometers as geomagnetic pulsations or magnetic pulsation events (MPEs) (Anderson et al., 1992a,b; Erlandson \& Anderson, 1996). Erlandson \& Ukhorskiy (2001) suggested that EMIC waves, in the equatorial regions, appear five times more often during geomagnetic storms than during quiet periods.

Magnetometers located on the Earth's surface register different types of waves. We can simply distinguish them, with the support of Fourier's numerical analysis, based on their dynamic power spectra (DPS, intensity shown in the frequency-time representation). EMIC waves, in the band of several $\mathrm{Hz}$, propagating through the magneto- and iono-sphere up to the Earth's surface are called continuous (Pc 1) or irregular (Pi 1) pulsations (Jacobs et al., 1963). They have specific signatures and are well recognized in DPS. Almost 160 years ago (in 1859), the first micropulsations of the Earth's magnetic field were recorded by B. Stewart (1861). It was during the largest geomagnetic storm on record, i.e. September 1-2, 1859. This storm is also known as the Carrington Event (Carrington, 1859). It caused significant magnetic field disturbances, very pronounced aurora displays and the destruction of telegraph systems. In those days observations were carried out with simple instruments that recorded the data on chart recorders. Magnetic pulsations can potentially have a significant impact on life on Earth. Some studies have shown that the Pc 1 pulsations can affect biological
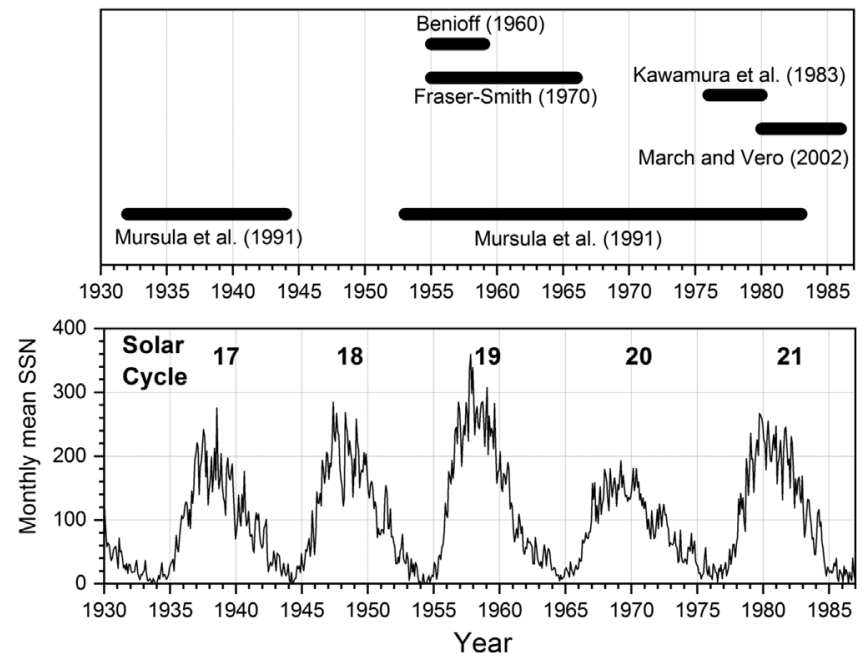

Fig. 1. Periods of time covered by respective study (top panel) and cycles of solar activity in terms of the SSN (bottom panel). Source of the SSN: WDC-SILSO, Royal Observatory of Belgium, Brussels.

systems (Subrahmanyam et al., 1985) and even cause the myocardial infarction in humans (Kleimenova et al., 2007). Therefore, their analysis of this point of view is very important.

The first statistical analysis of magnetic pulsations covered a time interval of 5 years (1955-1959) and was limited to the period of pulsation in the range of $0.3-120 \mathrm{~s}(0.008-$ $3.333 \mathrm{~Hz}$ ) (Benioff, 1960). The results showed a negative correlation between the occurrence rate of pulsations and the sunspot number (SSN). Similar conclusions also resulted from later observations (Fraser-Smith, 1970; Kawamura et al., 1983; Mursula et al., 1991, 1996; Märcz \& Verö, 2002) although several deviations from this trend were noted as well. The time ranges of these studies together with the SSN are displayed in Figure 1. The temporal and spatial characteristics of Pc 1 pulsations and their association to the EMIC waves were studied using ground-based (Kerttula et al., 2001; Guglielmi et al., 2006; Guglielmi \& Kangas, 2007; Bortnik et al., 2008) and satellite observations (Engebretson et al., 2008; Park et al., 2013). Nomura et al. (2011) presented their frequency-dependent polarization attributes and relation to the density of the $F$ region of the ionosphere. In this paper we revisit the question of the correlation between the detection rate of magnetic pulsations and the SSN and other indicators of solar activity. For this purpose, we used unique data obtained from a research station located in south-eastern Poland. Unlike previous studies, this observatory is located at medium geomagnetic latitudes (L-shell $\approx 2$ ). Almost continuous observations carried out at this station covered the period of 12 years (2005-2016). This allows us to study magnetic pulsations throughout the last 24th solar cycle (Zieba \& Nieckarz, 2014; Pesnell, 2016). This cycle is unique compared to several earlier cycles of solar activity. It is characterized by a long period of almost complete disappearance of solar activity and a low amplitude in the maximum phase. These differences are clearly visible in Figure 2. The paper is organised as follows. The data and data analysis methods used in this study are described in Sections 2 and 3, respectively. In Section 4 we present the results of our analysis. The discussion and conclusions are included in Section 5 and 6, respectively. 


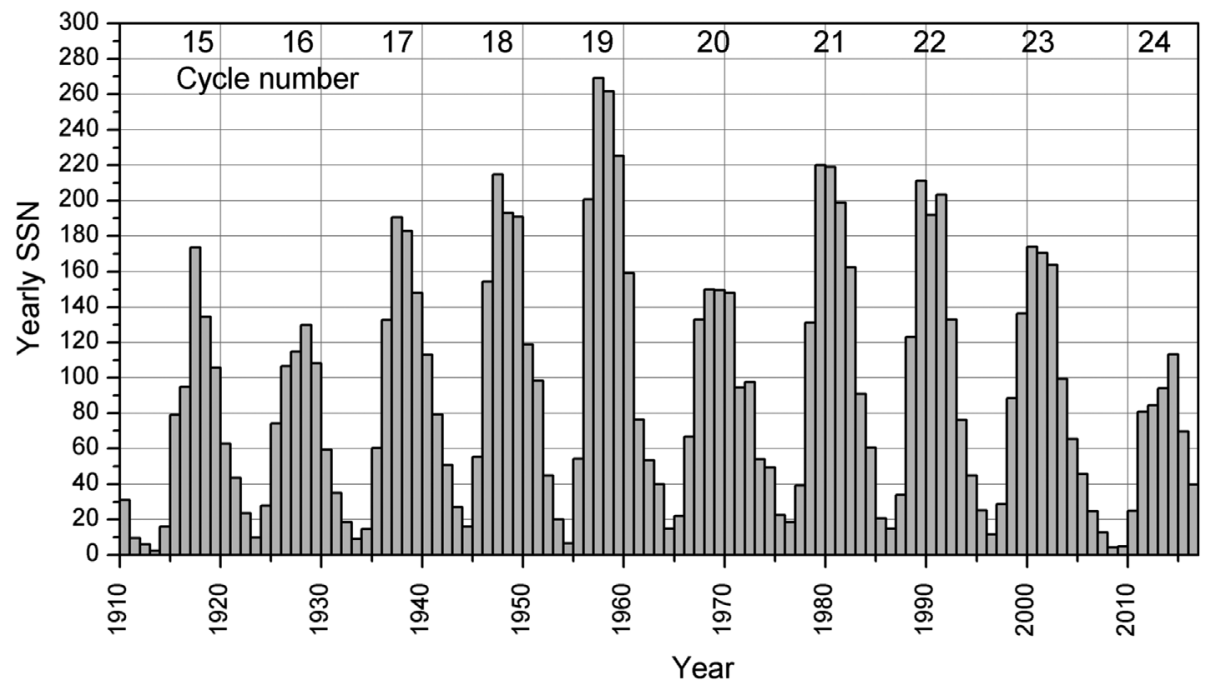

Fig. 2. Last 10 cycles of solar activity expressed in terms of the yearly SSN. Research carried out in this study cover the last cycle of solar activity seen in the figure. Source of the SSN: WDC-SILSO, Royal Observatory of Belgium, Brussels.

\section{Data}

Observations from the ELF Hylaty station are the basis of our current research. The station was constructed and maintained by Astronomical Observatory of the Jagiellonian University in Kraków, Poland. The station is located in the Bieszczady Mountains at the geographic location $49.2035^{\circ} \mathrm{N}$, $22.5438^{\circ} \mathrm{E}$ (L-shell $\approx 2.0$ ) near the Zatwarnica village (south-eastern Poland). The location of the ELF Hylaty station is shown in Figure 3. The station is placed far away from large human and industrial premises, power transmission lines and routes (motorways, railways) as well. High quality of collected data and low level of electromagnetic interference in the recorded ELF signal was confirmed in the analysis carried out by Nieckarz (2016). This allows us to carry out high-quality observations of magnetic waves for ultra-low and extremely low frequency (ULF/ELF, from 0.1 to $5 \mathrm{~Hz}$ ) ranges.

The main element of the receiver are two horizontal magnetic (coils) antennas directed in the NS and EW directions, respectively. The antennas are $100 \mathrm{~m}$ away from the underground receiver. A simplified block diagram showing the main elements of the measurement system is shown in Figure 4. This system enable us to record the signal with a sampling frequency of $175 \mathrm{~Hz}$ in the bandwidth $(3 \mathrm{~dB})$ from 0.03 up to $52 \mathrm{~Hz}$. The magnetic field components $B_{\mathrm{NS}}$ and $\mathrm{B}_{\mathrm{EW}}$ are recorded synchronously with an amplitude resolution of $3.8 \mathrm{nT}$ per 16 bits (58 fT per ADC level). The exact description of the station was presented in the publication by Kulak et al. (2014). The ELF "raw data" are collected by the Astronomical Observatory of the Jagiellonian University and are available for scientific purposes upon request to Cracow ELF Group (http://www.oa. uj.edu.pl/elf).

We analyzed the data sets recorded by the two antennas during the period of 12 years (2005-2016, 4383 days). Throughout this period, observations were not carried out, due to a technical malfunction, for only two months (May and June 2005). Sometimes also short data gaps appear, usually of the duration of about one day, due to periodic maintenance of the

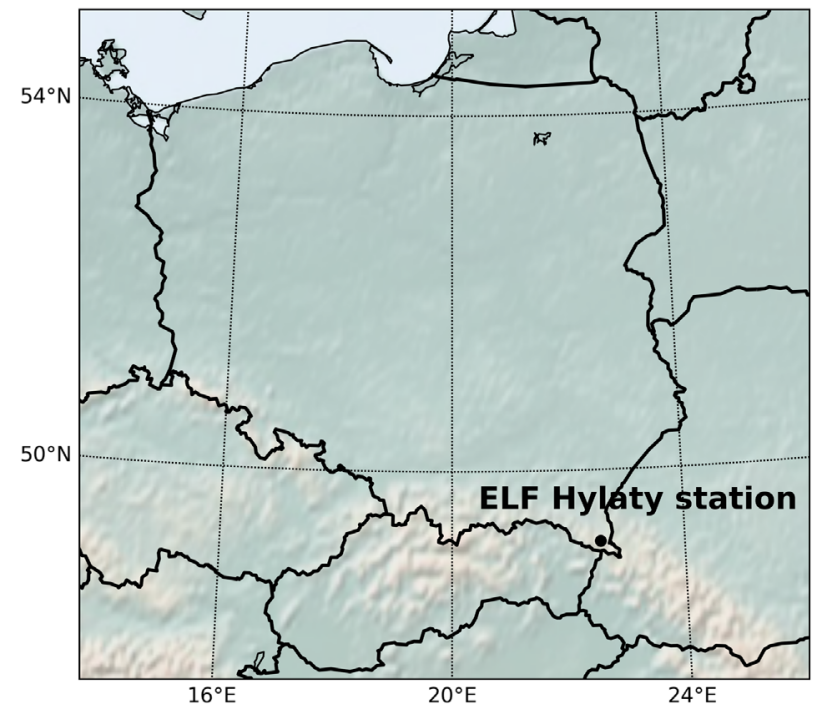

Fig. 3. Location of the ELF Hylaty station in south-eastern Poland.

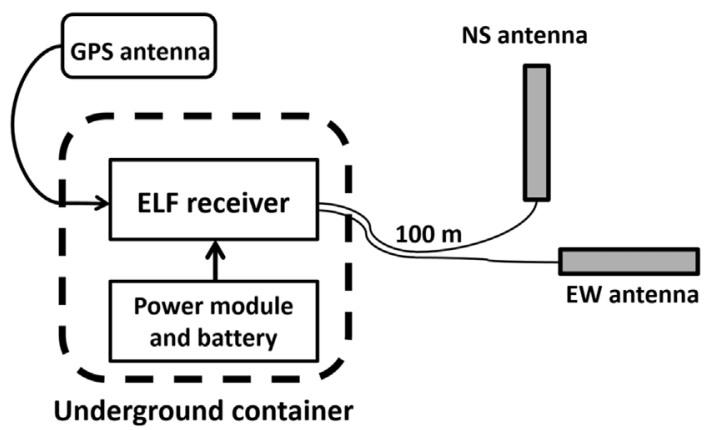

Fig. 4. A simplified block diagram of the Hylaty ELF station.

equipment. In the considered period of time, only $9.6 \%$ (8.3\% without taking these two idle months into account) of the observational time was affected by data gaps. 


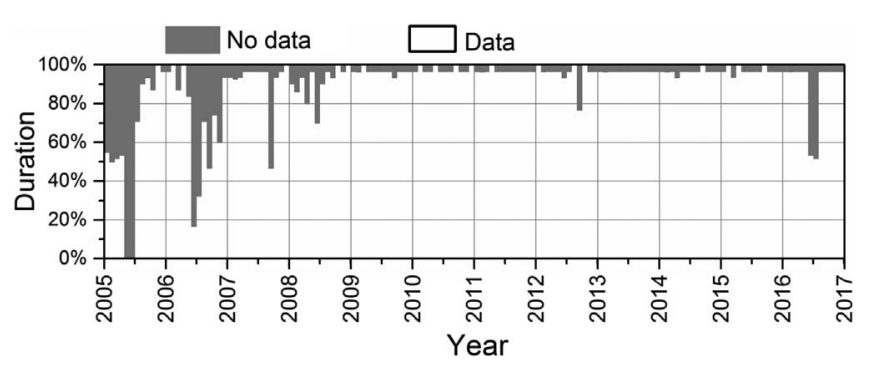

Fig. 5. Percentage of observation and idle time in the successive months (in the ELF Hylaty station). A white background shows the percentage of time when regular observations were made. Contrary, a gray background indicates the percentage of time when no observations were not carried out.

Figure 5 shows the percentage of observation time and idle time in the successive months. In addition to the observations from the ELF Hylaty station, in the present research, we used several databases containing observations from other instruments. These data were mainly related to various indicators characterizing the level of solar activity. We will briefly present each of these databases while discussing the results of our research in Section 4.

\section{Data analysis method}

The receiving system, presented above, provides "raw data", i.e. changes in the magnetic field recorded by individual antennas. To obtain the appropriate material for scientific analysis, these data were subject to Fourier analysis. In detail, every data set (separately for NS and EW antennas) was divided into non-overlapped sub-data sets of a time size $2^{14}=16384$ samples, equal to $93.6 \mathrm{~s}$ of observation. Consequently, this gives a frequency resolution of $0.01 \mathrm{~Hz}$ in Fourier analysis. Next, every data sample was individually detrended with a linear trend model. In this way, for every $24 \mathrm{~h}$ observations, $923(86400 \mathrm{~s} / 93.6 \mathrm{~s})$ steps in a time domain and 500 $(5.0 \mathrm{~Hz} / 0.01 \mathrm{~Hz})$ steps in frequency were created. The total DPS were calculated on the basis of signals from both (NS and EW) antennas. For the selected time window individual power spectra (PS) for each antenna $\left(\mathrm{PS}_{\mathrm{NS}}, \mathrm{PS}_{\mathrm{EW}}\right)$ were calculated using the Fast Fourier Transform (FFT) algorithm (where $\left.\mathrm{PS}=|\mathrm{FFT}|^{2}\right)$. Next, adding these power spectra $\left(\mathrm{PS}_{\mathrm{NS}}\right.$ and $\mathrm{PS}_{\mathrm{EW}}$ ) the total value of $\mathrm{PS}_{\mathrm{TOT}}$ was obtained. Finally, the permanent background noise which depends on the frequency was removed. For this purpose, the total power spectrum $\left(\mathrm{PS}_{\mathrm{TOT}}\right)$ is multiplied by frequency raised to the power of two $\left(f^{2}\right)$. This simple procedure causes the $\mathrm{PS}_{\mathrm{TOT}}$ to take a flat shape in the frequency range from 0.03 to $5 \mathrm{~Hz}$ (see Fig. 6). Figure 6 presents the hourly-averaged spectrum in the frequency range $(0.03-10 \mathrm{~Hz})$ with the background noise (top panel) and the noise removed (bottom panel). The measurements for the purpose of the figure were done on January 25, 2005 (6:0007:00 UTC). This figure also displays two characteristic structures corresponding to magnetic pulsations. The applied procedure makes it easier to recognize the characteristic features of pulsations appearing in the dynamic spectra.

In Figures 7 and 8 examples of magnetic pulsation signatures are presented. Figure 7 shows the total DPS (DPS ${ }_{\mathrm{TOT}}$ )
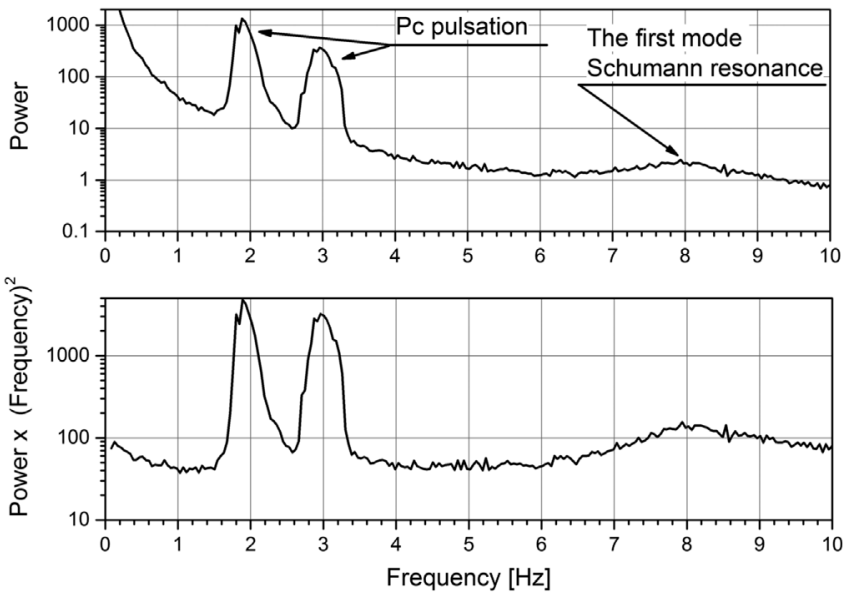

Fig. 6. The hourly averaged power spectrum of the signal registered by NS antenna of the ELF Hylaty station on January 25, 2005 (06:00-07:00 UTC, top panel) and the same power spectrum after reduction of a permanent noise shape $1 / f^{2}$ (bottom panel).
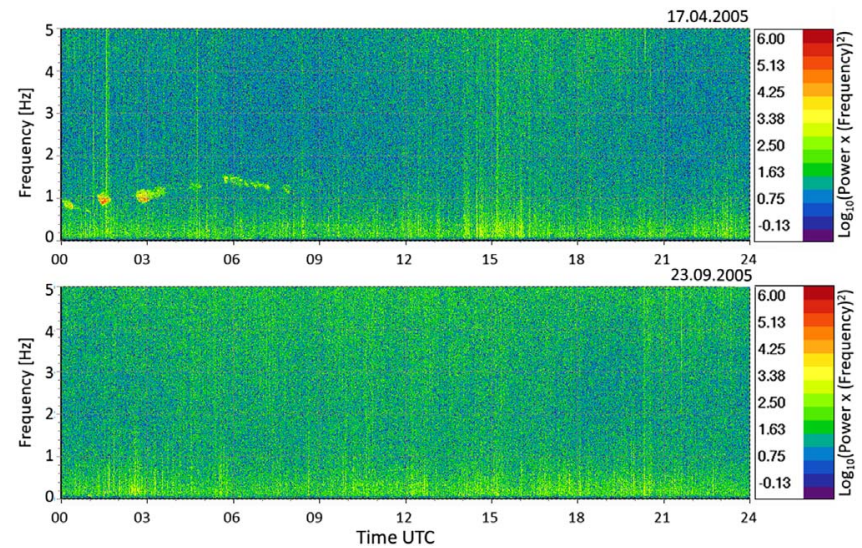

Fig. 7. Example of the total DPS for two separate days: 17.04.2005 (top panel) and 23.09.2005 (bottom panel). On April 17, 2005, clear signatures of pulsation from midnight up to 8 UT are observed.

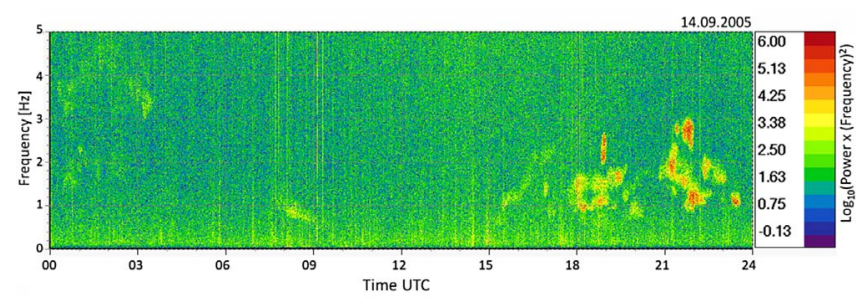

Fig. 8. Example of the total DPS on 14.09.2005. During this day, a very bright and long-lasting magnetic pulsation can be observed (between 18 and 24 UT).

for two separate days: 17.04.2005 (top panel) and 23.09.2005 (bottom panel). On April 17, 2005, clear signs of pulsation from midnight up to $8 \mathrm{UT}$ can be seen. This figure shows a typical dynamic spectrum of magnetic pulsations. While during the whole day of September 23, 2005 there are no signatures of magnetic pulsations at all (bottom panel). Sometimes pulsations 


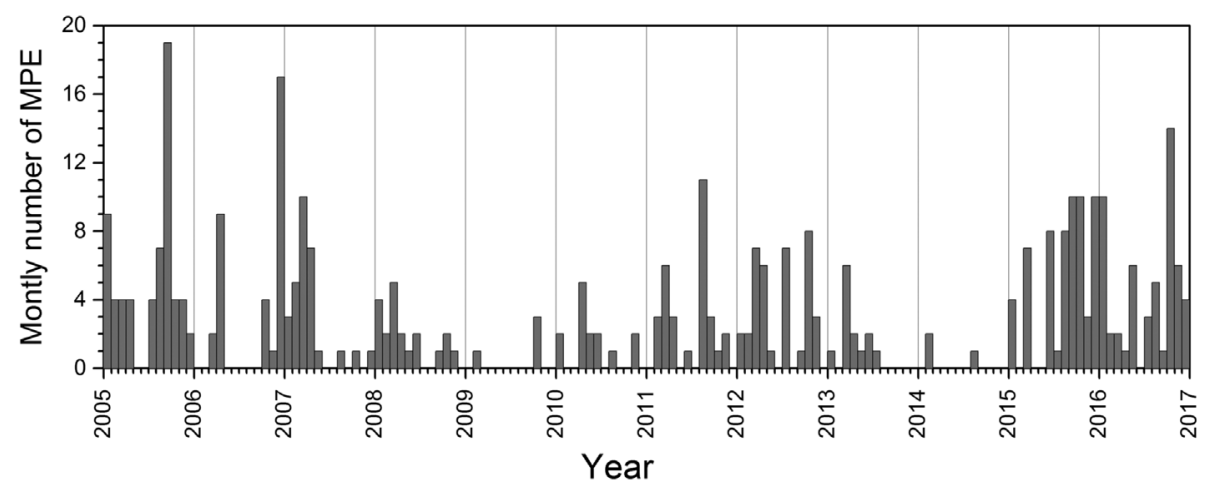

Fig. 9. Monthly distribution of the occurrence rate of magnetic pulsation events in the period 2005-2016.

can be very intense. An exceptional intense pulsation is shown in Figure 8. On September 14, 2005 a very bright and lasting the entire day magnetic pulsation was observed.

In our study, days with magnetic pulsation were identified by visual inspection of daily DPS $_{\mathrm{TOT}}$. Such daily visual identification of pulsations requires about one minute. This technique seems to be effective and has been commonly used (Posch et al., 2010; Mergu \& Dixit, 2011; Paulson et al., 2017). The identification of magnetic pulsation was carried out only by one observer. A pulsation event was considered to occur if, in DPS $_{\text {TOT }}$ image, it is clearly distinguishable from the background (by color change and size). In addition, it must have also a spot shape and size larger (around two times) than the size of the statistical background fluctuations. Each daily DPS TOT $_{\text {was }}$ carefully examined twice. Magnetic pulsations are definitely considered true if visual inspection confirmed their appearance twice. However, it should be noted that using this method we may overlooked some faint MPEs. The procedure described above allowed us to create a list of all pulsations observed by the ELF Hylaty stations in the period 2005-2016. This data set was used as basis for our statistical analysis carried out in the next Section.

\section{Results}

Having almost continuous, high quality and long-term observations of magnetic pulsations at middle geomagnetic latitudes, we could analyze their relationship with solar activity. The results of this analysis are presented in the following subsections.

\subsection{Occurrence rate of magnetic pulsation}

For the considered period of time we were able to obtain 3961 daily dynamic spectra. The magnetic pulsation signatures were found in 360 daily spectrograms. This corresponds to $9.1 \%$ of all daily spectrograms. The monthly distributions of MPEs can be seen in Figure 9. At first glance this distribution looks quite randomly-distributed, and it is difficult to recognize the occurrence of any characteristic pattern. We can only distinguish periods of several months when there are more MPEs separated by periods when MPEs are not observed at all. The highest number of MPEs per month (19) was recorded in September 2005, while the longest period in which MPEs were not recorded at all covered seven months from March to September 2009. A similar period of a very low rate of MPEs appeared in 2014. This is intriguing because the first of these periods coincided with a clear minimum of solar activity and the second one with a period of high solar activity.

\subsection{Occurrence rate of MPEs and sunspots}

The most popular indicator of solar activity is the SSN, which reflects the number of sunspots simultaneously visible on the solar disk during a given period of time. Therefore the MPEs detection rate has been mostly compared to the SSN. Figure 10 presents the yearly distribution of occurrence rate of SSN (top panel) and MPEs (bottom panel) in the considered period of time. The yearly binarization makes it easier to recognize trends emerging in the MPE detection rate. The obtained distributions do not allow to find significant correlations between both phenomena (i.e. a Pearson correlation coefficient of $R=0.02$ ). From the figure it can be seen that a significant increase in the detection rate of MPEs is observed in the declining phase of the 23rd cycle (2005-2007), in the growth phase of the 24th cycle (2011-2012) and in the decay phase of the 24th cycle (2015-2016). The negligible amount of MPEs was recorded during the period of very low solar activity (20092010) and, surprisingly, during the maximum phase of the 24th cycle (2013-2014). The highest occurrence rate of MPEs (61) has been recorded in 2005 and 2015. Only six less magnetic pulsations were recorded in 2016. It seems that the most significant differences between the considered distributions appear in the declining phases of solar cycle.

\subsection{Occurrence rate of MPEs and CMEs}

Sunspots are a phenomenon observed in the solar photosphere. Their presence indicates solar activity but they have no direct impact on the Earth. Only fast magnetized plasma escaping from the Sun (CMEs or high-speed solar wind streams originated from coronal holes) can generate geomagnetic disturbances (Cranmer, 2002, 2009; Cranmer et al., 2017). The most significant magnetic storms are caused by CMEs (Richardson et al., 2006; Zhang et al., 2007). Therefore, it is important to consider the relation between CMEs and MPEs. CMEs recorded by $\mathrm{SOHO} / \mathrm{LASCO}$ instrument are included among others in the SOHO/LASCO CME catalog (https://cdaw.gsfc.nasa.gov/ CME_list/). This catalog contains, together with basic kinematic 

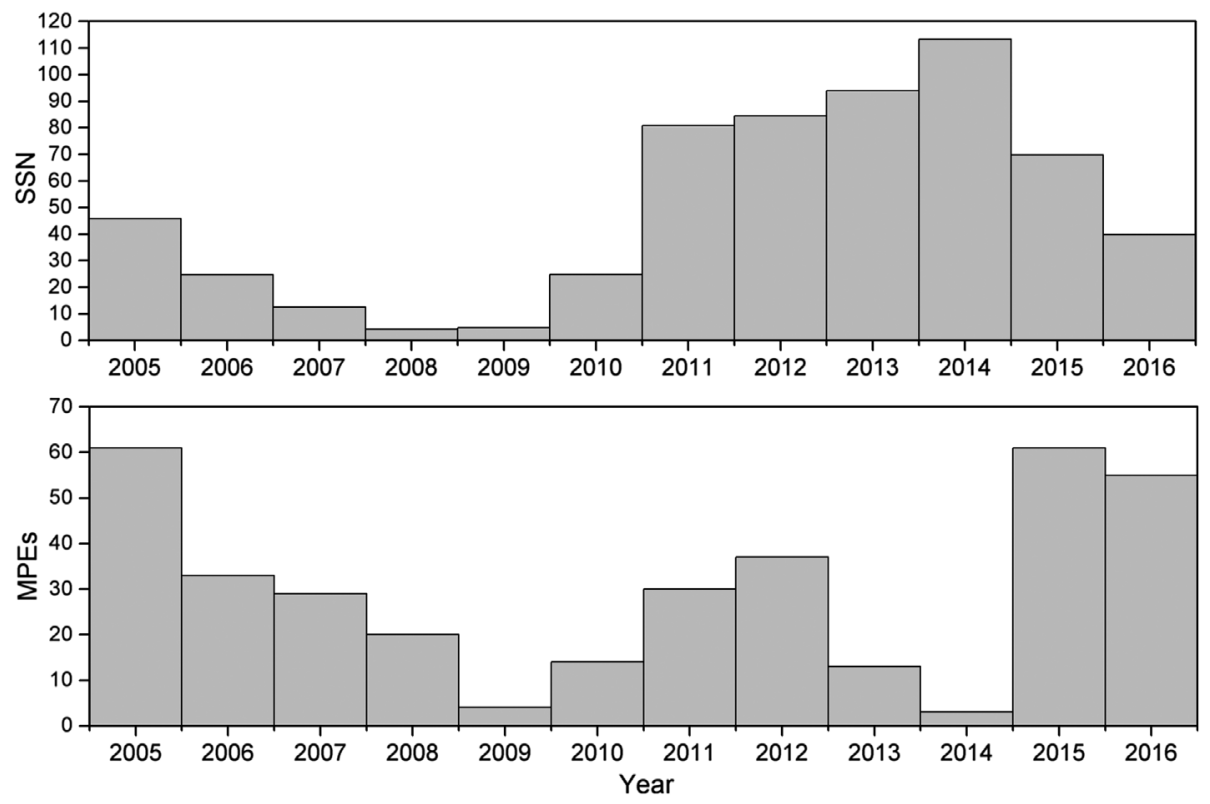

Fig. 10. The yearly distribution of occurrence rate of SSN (top panel) and MPEs (bottom panel) in the period of 2005-2016.

parameters, all CMEs registered since 1996 (Yashiro et al., 2004). In addition to the data characterizing CMEs, in our research we used UV movies from EIT (https://lasco-www. nrl.navy.mil), SDO (https://sdo.gsfc.nasa.gov) and plots of Dst (http://wdc.kugi.kyoto-u.ac.jp) included in the CDAW Data Center (https://cdaw.gsfc.nasa.gov).

Figure 11 shows the distribution of the detection rate of all the CME (18 025) included in the SOHO/LASCO CME catalog in the period of time 2005-2016. These distributions very closely follow the SSN, as confirmed by the very high correlation coefficient between these parameters $(R=+0.91$ at significance level $p<0.05)$. This means that, similar as we observed for the SSN (Fig. 10), the detection rate of CME is not significantly correlated with the number of MPEs. The largest occurrence rate of CMEs was observed in 2014 when the number of MPEs was negligible. The correlation coefficient $(R=-0.18)$ between the detection rate of CMEs and MPEs indicates very poor anticorrelation between these parameters. This result seems quite surprising, as it implies that solar activity does not significantly affect the MPE generation.

However, we must mention here that the 24th cycle of solar activity is significantly different from previous cycles. At the minimum and maximum of 24th cycle of solar activity, many very poor bursts (narrow and faint) were observed (Michalek et al., 2019). In this period of time the amount of CME does not reflect properly the magnetic activity of the Sun (see Fig. 10). This enhanced rate of CME in solar cycle 24 occurred due to anomalous condition in the solar corona and in the interplanetary medium. Due to a very long period of decay of solar activity (2008-2010) the interplanetary medium has become extremely rarefied which greatly facilitated the generation and expansion of CMEs (Gopalswamy et al., 2015, 2016; Michalek et al., 2019).

Therefore it seems more reasonable to study the correlations that occur between potentially geoeffective CMEs and MPEs. Potentially geoeffectve CMEs are generally very energetic so

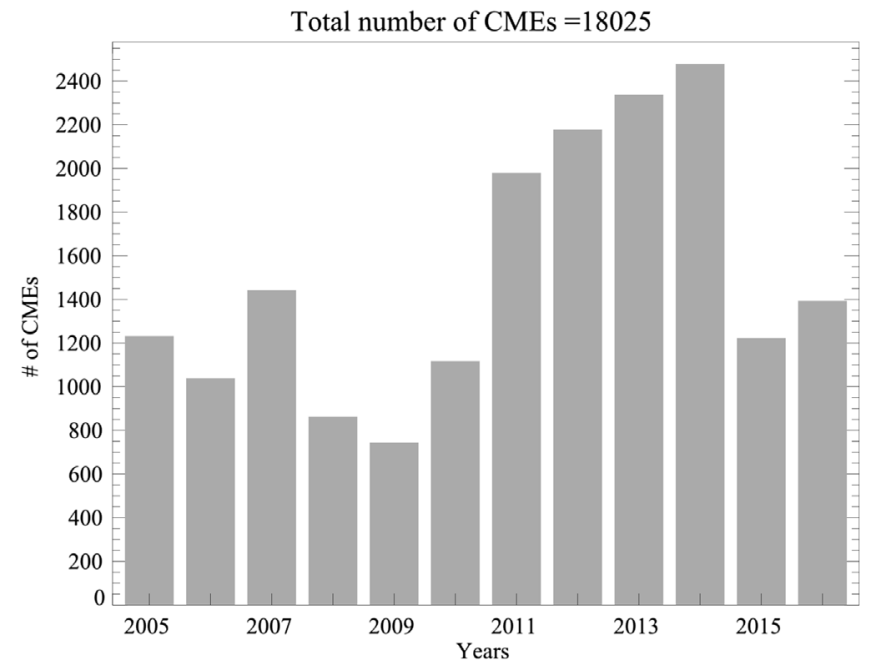

Fig. 11. Distribution of detection rate of all CME (18025) included in the SOHO/LASCO CME catalog in the period of time 2005-2016.

they should have high speeds and large angular widths (e.g. Richardson et al., 2001; Zhang et al., 2003; Gopalswamy et al., 2005). Figure 12 shows the distribution of the detection rate of potentially geoeffective CMEs in the period of time 2005-2016. In this work, we assume that potentially geoeffective CMEs should have an apparent speed higher than $500 \mathrm{~km} \mathrm{~s}^{-1}$ and apparent angular width larger than 100 degrees as visible in LASCO coronagraphs. It is necessary to mention that single coronagraph observations are strongly affected by projection effects (Temmer et al., 2009; Shen et al., 2013; Balmaceda et al., 2018). Therefore the basic attributes of CMEs (speed, width, acceleration) are provided in the plane-of-sky.

As can be seen from the figure, this did not solve our issue. The correlation between the detection rates of CMEs and MPEs still remains negligible $(R=-0.08)$. 


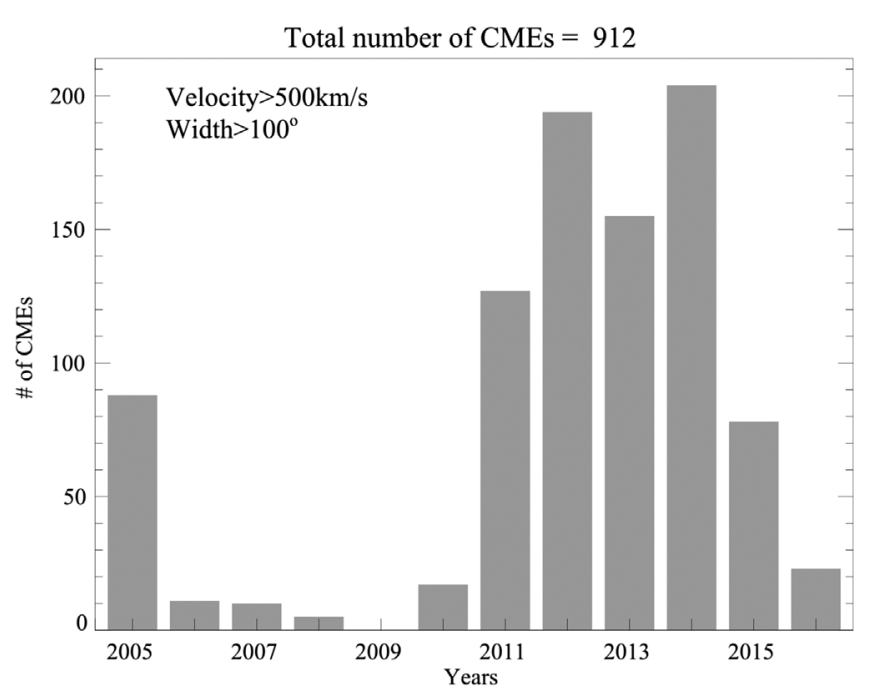

Fig. 12. Distribution of detection rate of potentially goeffective CMEs in the period of time 2005-2016.

In conclusion, a simple analysis based on the basic attributes of CMEs is not sufficient to assess the solar sources generating the observed MPEs. Therefore, in the next section we investigate MPEs association to other geoeffective phenomena observed at Earth.

\subsection{Occurrence rate of MPEs and Dst index}

The most popular indicator determining the intensity of geomagnetic disturbances is the Disturbance Storm Time Index $(D s t)$. The Dst index measures the intensity of the equatorial ring current. It is derived from a network of four near-equatorial geomagnetic observatories. A negative Dst value means that Earth's global magnetic field is weakened. This is particularly the case during geomagnetic storms when the intensity of ring current increases. The Dst index is mostly determined by solar activity. The coupling with the Sun is via the solar wind, which carries solar transients from the Sun to the Earth. Therefore the Dst index primarily responds to the conditions of the solar wind impinging on Earth, and therefore any disturbance in the Dst index can be ultimately related to phenomena of solar origin (CMEs or fast solar wind from coronal holes). Earlier studies have indicated that magnetic pulsations can be observed during and after geomagnetic storm but statistically it tends to occur 3-7 days after the commencement of magnetic storms and even from 4 to 10 days following large magnetic storms (Wentworth, 1964; Campbell \& Stiltner, 1965; Heacock \& Kivinen, 1972; Engebretson et al., 2008). Since 1957, the Dst index has been maintained by the World Data Center for Geomagnetism Kyoto and National Centers for Environmental Information (formerly NGDC).

Having hourly values of $D s t$ index (Dst $\mathrm{h}_{\mathrm{h}}$, http://wdc.kugi. kyoto-u.ac.jp) we were able to determine, for a given day, an average daily $D s t$ index $\left(D s t_{\mathrm{d}}\right)$, its variance $\left(D s t_{\mathrm{var}}\right)$, its daily variability $\left(D s t_{\mathrm{ptp}}\right)$ which is a daily range of $D s t$ index and daily minimum $\left(D s t_{\mathrm{min}}\right)$. Statistical analysis of these parameters of Dst index revealed that both these phenomena (MPEs and $D s t)$ are correlated with each other. The Dst index does not have Gaussian distribution, therefore for its analysis we used nonparametric tests (Kolmogorov-Smirnov and Mann-Whitney $\mathrm{U}$ tests). These tests were employed to show a significance in differences of the Dst index in days when MPEs are observed or not. Both tests indicated that the distribution of the Dst values varies significantly $(p<0.001)$ between days with observed MPEs and without MPEs. The $D s t_{\mathrm{d}}$ is significantly lower in days with observed MPEs compared to days without MPEs and is equal -20.7 and -9.3 (median is equal -16.1 and -7.0 , respectively), respectively. Similarly significant differences was obtain for $D s t_{\mathrm{var}}, D s t_{\mathrm{ptp}}$ and $D s t_{\mathrm{min}}$. The mean values of the $D s t_{\mathrm{var}}\left(D s t_{\mathrm{ptp}}, D s t_{\mathrm{min}}\right)$ distribution for the days with MPEs and without MPEs were $122.3(29.6,-36.4)$ and 56.2 (20.9, - 19.9), respectively.

Next we calculated $\Delta D s t_{\mathrm{d}}$ as absolute difference between the mean $D s t_{\mathrm{d}}$ for days with observed MPEs and without MPEs. Then we found optimal time delay equal to -3 days where value of $\Delta D s t_{\mathrm{d}}$ is maximum as function the time delay between MPE and used Dst index. Calculations were made in range from -5 to 0 days. We assuming that MPEs are delayed after main phases of geomagnetic storms. Identical calculations and time delay analysis were made for others indices $\left(\Delta D s t_{\mathrm{var}}, \Delta D s t_{\mathrm{ptp}}\right.$, $\left.\Delta D s t_{\text {min }}\right)$ and the same result was obtained. The significantly optimal time delay equal to -3 days was obtained where $D s t_{\mathrm{d}}$ in days with observed MPEs compared to days without MPEs is equal -24.8 and -8.8 respectively, and $D s t_{\mathrm{var}}, D s t_{\mathrm{ptp}}$, $D s t_{\min }$ are equal $187.3,32.5,-41.9$ and $49.5,20.5,-19.2$ respectively.

For each month we also calculated the number of days with $D s t_{\text {min }}$ index lower than $-30,-40,-50$ and $-60 \mathrm{nT}$, respectively. We found the most significant correlation $(R=+0.49$, $p<0.05)$ between the detection rate of MPEs and geomagnetic disturbances with the $D s t_{\min }<-40 \mathrm{nT}$. The analysis presented here clearly indicates that MPEs are significantly associated with geomagnetic storms disturbances and therefore their source must be at the Sun.

To ultimately confirm the above thesis, in Figure 13 we show yearly distributions of numbers of days with MPEs, $D s t_{\min }<-40 \mathrm{nT}, D s t_{\min }<-50 \mathrm{nT}, D s t_{\min }<-60 \mathrm{nT}, D s t_{\min }$ $<-70 \mathrm{nT}$, and $D s t_{\min }<-100 \mathrm{nT}$, respectively. The presented distributions clearly show that MPEs are significantly correlated with the moderate $\left(D s t_{\min }<-70 \mathrm{nT}, R=0.72\right)$ and intense geomagnetic disturbances $\left(D s t_{\min }<-100 \mathrm{nT}, R=0.70\right)$. We must emphasize that the occurrence rate of MPEs in the maximum phase of the 24th solar cycle tracks the geomagnetic disturbances with $D s t_{\min }<-70 \mathrm{nT}$ and $D s t_{\min }<-100 \mathrm{nT}$ very well. The significance of these correlations has been confirmed by appropriate statistical tests. It is worth reminding here that CMEs are not the only source of geomagnetic disturbances. MPEs and geomagnetic storms, especially the moderate ones, can be generated by co-rotating interaction regions as well.

\subsection{Energetic CMEs and MPEs}

The purpose of our research was to identify the solar sources of MPEs recorded by the ELF Hylaty station. The issue doesn't seem to be that simple. The use of basic indicators of solar activity for this purpose did not bring any positive result. We conducted our research mainly during the 24th solar cycle. As we mentioned above, this cycle was unique in many aspects. During the solar cycle 24 we observed a significant increase of the $\mathrm{CME}$ rate compared to the solar cycle 23 , even though the 

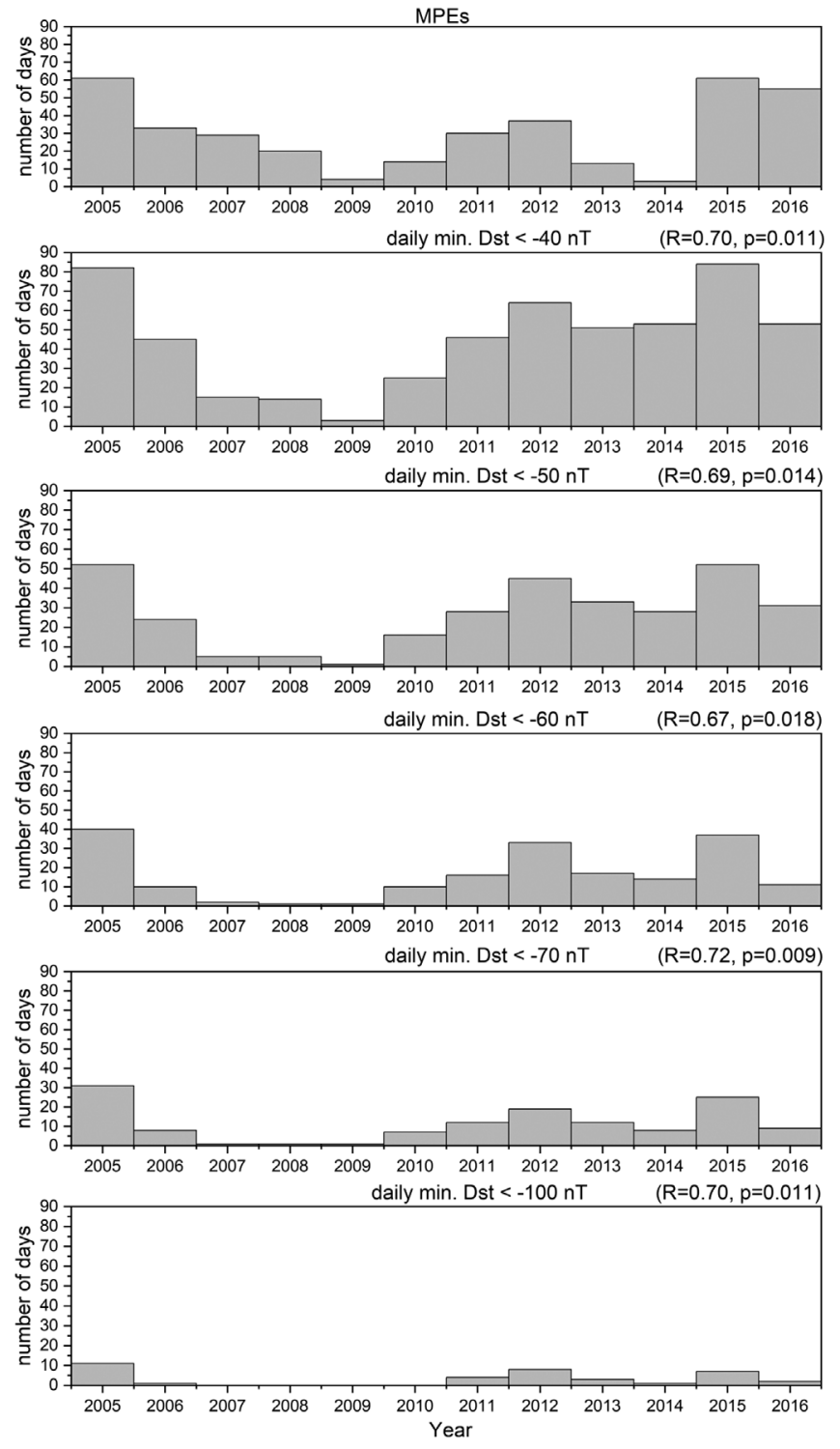

Fig. 13. Figure, from top to bottom, shows yearly distributions of numbers of days with MPEs, Dst $t_{\min }<-40 \mathrm{nT}$, Dst $\mathrm{min}_{\min }<-50 \mathrm{nT}$, Dst $_{\text {min }}<-60 \mathrm{nT}$, Dst $t_{\min }<-70 \mathrm{nT}$, and Dst $\mathrm{min}_{\min }<-100 \mathrm{nT}$, respectively. At the top of the panels, the correlation coefficients between the respective MPEs and Dst distributions are presented.

sunspot number has been smaller by $40 \%$ (Wang \& Colaninno, 2014; Webb et al., 2017). This discrepancy is also observed for wide (width $>30^{\circ}$ ) (Petrie, 2015a) and halo events (Gopalswamy et al., 2015). What is important, during the rising phase of solar cycle 24 , this discrepancy was proved by a different method of CME detection (Petrie, 2015b).

Luhmann et al. (2013) and Petrie (2013) suggested that this anomalous increase in the CME rate is due to the weak solar polar magnetic field during the extended solar cycle 23/24 minimum. Gopalswamy et al. (2014a, 2015) suggested that this discrepancy is due to the significant decline in total (magnetic+plasma) pressure during this last minimum of solar activity. It is supposed that the complex magnetic field structure together with the low total ambient pressure are responsible for

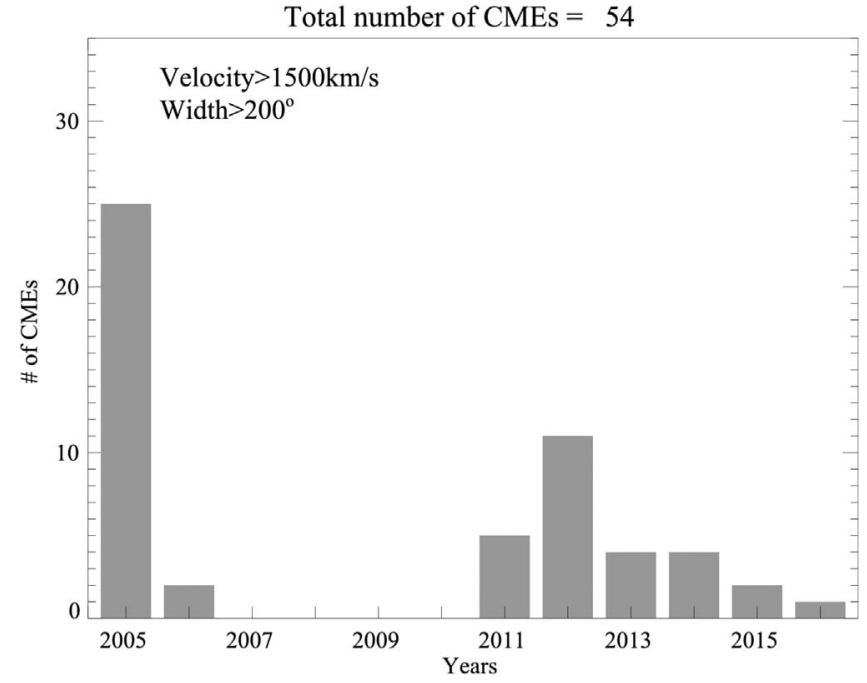

Fig. 14. Distribution of detection rate of the most energetic CMEs $\left(V>1500 \mathrm{~km} \mathrm{~s}^{-1}\right.$, angular width $\left.>200^{\circ}\right)$ in the period of time 20052016.

the significant increase of CME detection rate in the last solar cycle (Michalek et al., 2019). In a low-pressure environment, CMEs can easily erupt and then expand. Due to the decline of coronal and heliospheric total pressure the CMEs during the last solar maximum are on average wider compared to these during the maximum of solar cycle 23. So they are also rarefied and therefore less geoeffective. This means that, on average, all the CMEs in the two considered solar cycles are different. Hence geomagnetic activity in cycle 24 is low. Watari (2017) has recently made a comprehensive data analysis of geomagnetic activities in the rising phase of the 24th cycle (20092015). It was revealed that geomagnetic activity in the considered period of time was at the lowest level since 1957. There were only 17 severe geomagnetic storms $\left(D s t_{\min }<-100 \mathrm{nT}\right)$ caused mostly by halo and partial halo CMEs. Only two of them were due to large coronal holes. Therefore, in the last cycle, only exceptionally energetic CMEs generated intense geomagnetic disturbances. Figure 14 shows the distribution of detection rate for the most energetic CMEs $\left(V>1500 \mathrm{~km} \mathrm{~s}^{-1}\right.$, angular width $>200^{\circ}$-partial halo). By comparing Figure 14 with Figure 10, we can see a great convergence of this distribution with that for MPEs (Fig. 10, bottom panel, the correlation coefficient is 0.61 , significant according to the performed test). The most significant inaccuracy between these distributions appears in 2016. Then we observe a large number of MPEs not related to energetic CMEs. It is clear that geomagnetic storms, especially the most intense ones $\left(D s t_{\min }<-100 \mathrm{nT}\right)$, are due to CMEs. However, coronal holes are the most common source of the moderate disturbances $\left(D s t_{\min }>-100 \mathrm{nT}\right)$. Their relation with MPEs is discussed in the next section. We also analyzed the basic kinematic parameters of CME causing MPEs. Figure 15 displays the distributions of basic kinematic parameter (related to storm intensities) of CMEs. As we could have expected only very energetic CMEs can generate MPEs. The average speed of these CMEs is over $1100 \mathrm{~km} \mathrm{~s}^{-1}$. While, the average speed of all recorded CMEs is only about $400 \mathrm{~km} \mathrm{~s}^{-1}$. Only in two cases, the speed of CMEs determined in the LASCO field of view of was below $300 \mathrm{~km} \mathrm{~s}^{-1}$. In situ 

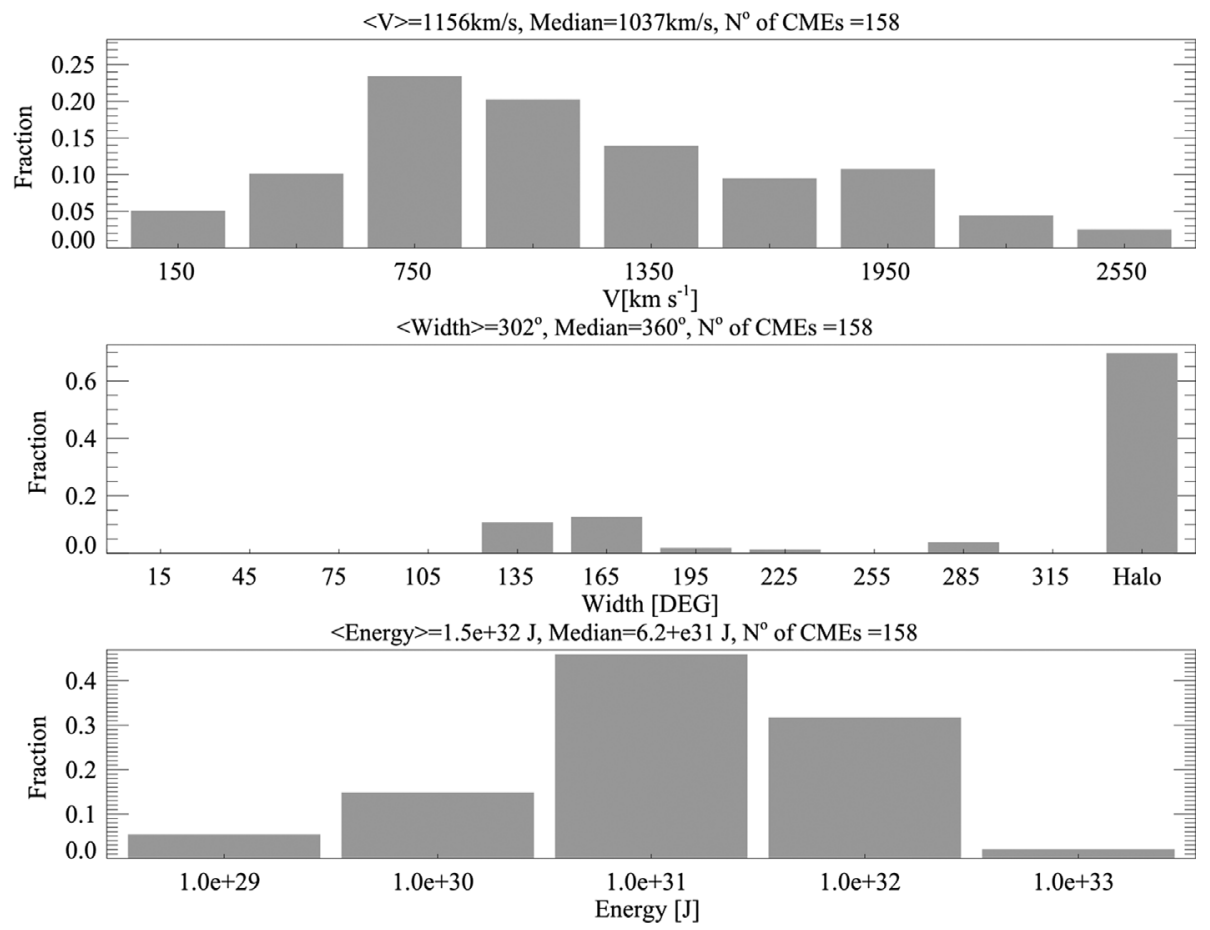

Fig. 15. Distributions of the basic attributed of CMEs associated with MPEs in the period of time 2005-2016. In successive panels we display the distributions of velocity, width and kinematic energy of these events.

observations of their interplanetary magnetic clouds confirmed this. Almost $70 \%$ of CMEs generating MPEs were halo events. The remaining $30 \%$ are very wide partial halo events (with angular width $>120^{\circ}$ ). The average kinetic energy of these CMEs is also very large and is equal to $1.5 \mathrm{e}+32 \mathrm{~J}$.

\subsection{Co-rotating interaction regions and MPEs}

Coronal holes have magnetic field open to interplanetary space and hence they are sources of fast solar wind. Thus, the coronal holes located near the Sun's central region are known to be the most geoeffective ones (Kim et al., 2005; Moon et al., 2005). The most intense geomagnetic storm, caused by a high-speed stream generated by a coronal hole during solar cycle 24 was observed on $07.10 .2015\left(D s t_{\min }=-124 \mathrm{nT}\right.$, Navia et al., 2018). In the previous section we showed that MPEs can be caused only by the most energetic CMEs. However, we observe a large sample of MPEs not associated with CMEs. Therefore, for a full understanding of this phenomenon, we need to determine all the solar sources of MPEs considered in this study. It can be assumed that these MPEs can be generated by fast (CIRs) or slow solar wind (Richardson \& Cane, 2012a, b). CIRs are compression regions ahead of coronal holes high-speed stream. They form due to the interaction of slower, ambient solar wind ahead of the higher-speed stream originating from the more persistent coronal holes. CIRs like CMEs can hit the Earth's magnetosphere, causing global geomagnetic disturbances.

To identify sources of the CIRs we employed data included in the SOHO/LASCO CME catalog (Gopalswamy et al., 2009). For this purpose, we use daily movies showing the Sun in UV (EIT/SOHO or AIA/SDO). In these images we can distinguish $\mathrm{CHs}$ (equatorial $\mathrm{CHs}$ on the west side of solar disk) potentially responsible for the generation of the geomagnetic disturbances (Norton et al., 1999; Akiyama et al., 2013). This catalog, apart from the basic attributed of CMEs, contains data from many available satellites and observatories focused on space weather research. Using this data (UV images and Dst profiles) we recognized all the coronal hole associated with geomagnetic storms during which the MPEs were also registered. During the decay phase of the 24 solar cycle (2015-2016) several very significant equatorial coronal holes appeared. They were responsible for moderate geomagnetic disturbances recorded in this period. This was especially observed in 2016, when we recorded only moderate geomagnetic storms mostly related to the equatorial coronal holes. It turned out that most MPEs were caused by CIRs (202 cases) compared to that generated by the energetic CMEs (158 cases).

Plotnikov et al. (2016), using Heliospheric Cataloguing, Analysis and Techniques Service (HELCATS), presented a statistical analysis of CIRs in the period from 2007 to 2014 (see Fig. 3 in Plotnikov et al., 2016). This period almost coincides with our research. Their observations were based on the Heliospheric Imager (HI) instruments on board STEREO spacecraft's (Eyles et al., 2009). They provide white-light images of the solar wind outflow from $\sim 15 R_{\odot}$ from the Sun out to $1 \mathrm{AU}$, and beyond. This enables them to study the CIRs and what is very important only CMEs directed and expanding to the Earth which are potentially geoeffective. Over the period under consideration (2007-2014), they were able to identify only 190 CIRs, while at the same time about 5 times more potentially geoeffective CMEs were observed (Plotnikov et al., 2016). By comparing this data with our results (the number of MPEs caused by CMEs and CIRs) we can conclude that CIRs do play an important role in the generation of MPEs. 

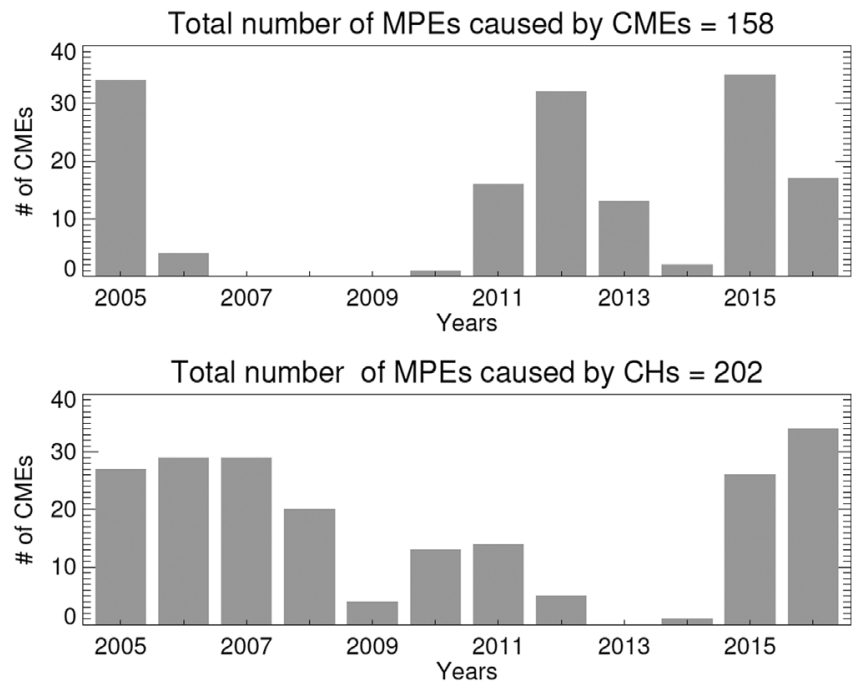

Fig. 16. The yearly distribution of occurrence rate of CMEs (top panel) and solar coronal holes (bottom panel) associated with MPEs during the period of time 2005-2016.

Figure 16 shows the yearly distribution of occurrence rate of CMEs (top panel) and solar coronal holes (bottom panel) associated with MPEs considered in this study.

As we see, only $44 \%$ of the MPEs were associated with storms caused by CMEs listed in the CDAW LASCO CME catalog. $56 \%$ of the MPEs were associated with storms caused by other phenomena including corotating interaction regions (CIRs), slow solar wind (Richardson \& Cane, 2012a, b) or CMEs not detected by LASCO. CMEs mostly generate MPEs in the maximum phase of the solar cycle. The significance of a given phenomenon depends on the phase of the solar activity cycle.

\section{Discussion}

Our study has shown that MPEs are mostly generated during moderate and intense geomagnetic storms. During such storms several plasma regions are affected in the magnetosphere and the whole current system of the magnetosphere and ionosphere is intensified. The main process which determines the transfer of the energy from the solar wind to the magnetosphere is magnetic reconnection. It significantly depends on the intensity of the southward oriented magnetic field $\left(B_{Z}\right)$ included in solar wind (Gonzalez et al., 1999).

The intense southward magnetic field can be included in CMEs and CIRs. Since, CIRs and CMEs have different morphology, duration and temporal profiles with respect to $\mathrm{B}_{\mathrm{Z}}$ they generate different storms. The most severe geomagnetic storms $\left(D s t_{\min }<-100 \mathrm{nT}\right)$ are caused by CMEs, especially by halo events (Zhang et al., 2007; Gopalswamy, 2009). That is why we are not surprised that they are closely related to MPEs. They mostly appear during the maximum phase of solar cycles. While during the descending and minimum phase of solar cycle coronal holes occur more frequently. CIRs are related to weak and moderate storms $\left(D s t_{\min }>-100 \mathrm{nT}\right)$ (Cramer et al., 2013).
It is intriguing why so many MPEs were associated with CIRs. Although the CIR-driven storms are less intense they can last much longer than those generated by CMEs. Thus, they can transfer a large amount of energy to the magnetosphere. CMEs and CIRs generally appear in different phases of solar cycles. MPEs are strongly related to both of these phenomena, therefore their occurrence rate cannot be associated with any phase of the solar cycle or with any indicator characterizing a 11-year sun cycle.

The very interesting result of our research is the fact that more MPEs were associated with CIRs than with CMEs. We revealed that only very energetic CMEs can generate MPEs. Our analysis was carried out mostly in solar cycle 24 which was anomalous in many aspects. It was significantly weaker, regarding the SSN, compared to a few previous solar cycle. Additionally, due to long duration of the last solar cycle and extremely low its activity, the interplanetary medium became very rarefied. The weak state of the heliosphere in cycle 24 allowed the CMEs to easily erupt and expand (Gopalswamy et al., 2014b, 2015). That is why during the last cycle the CMEs were observed much more frequently than during the previous one. However, their internal structure was also rarefied, so they did not pose a significant threat to our magnetosphere. Hence geomagnetic activity in this cycle was in the lowest level in the recent six solar cycles since 1957 (Watari, 2017). This effect can simply explain the fact that in cycle 24 more MPEs were caused by CIRs than by CMEs. This tendency depends on solar activity and can be reversed in other solar cycles. A longer series of observations is needed to fully explain relationship between MPEs and these both phenomena.

It is important to note that MPEs appeared mostly during recovery phases of the associated geomagnetic storms, frequently even at the end of this phase (Guarnieri et al., 2006). Because the recovery phase of a geomagnetic storm can last several days (especially in the case of CIRs) many MPEs were generated by the same CME or CIR. In our study the delay time is equal to in average 3 days after onset, when the magnetosphere is sufficiently energized (Kanekal, 2006; Engebretson et al., 2008).

\section{Conclusions}

In the present study the MPE (in the frequency range from 0.1 to $5 \mathrm{~Hz}$ ) occurrence rate recorded in the ELF Hylaty station located in the Bieszczady Mountains in Poland was studied. This station provided almost continuous and high-quality observations in a period of 12 years (2005-2016), covering almost the entire 24th cycle of solar activity.

In Section 4.2, we showed that in the case of magnetic observations made at medium geomagnetic latitudes $(\mathrm{L}$-shell $\approx 2$ ) there is no significant correlation between the occurrence rate of MPEs and the SSN. A similar conclusion was obtained for all the CMEs included in the SOHO/LASCO CME catalog (Sect. 4.3).

On the other hand, we showed a clear correlation between the occurrence rate of MPE and Dst index (Sect. 4.3), which reflects the global disturbance of the Earth's magnetic field. This clearly confirms that MPEs have their source at the Sun. It is also worth mentioning that our observations showed that MPEs 
appear much later than the Dst index minima caused by associated geomagnetic storms (about 3 days later).

Considering only the most energetic CMEs $\left(V>1500 \mathrm{~km} \mathrm{~s}^{-1}\right.$, angular width $\left.>200^{\circ}\right)$ (in Sect. 4.5), we found a good relation (correlation coefficient is 0.61) with the occurrence rate of MPEs (Fig. 14). However, to get even better agreement between the occurrence rate of MPEs and solar activity we need to take into account geomagnetic disturbances generated not only by CMEs but also by fast solar wind from equatorial coronal holes. The very interesting result of our research is the fact that more MPEs were associated with CIRs than with CMEs. Our analysis was carried out mostly in solar cycle 24 which was anomalous in many aspects. Due to elongated and extremely low last solar minimum the interplanetary medium became very rarefied allowing the CMEs to easily erupt and expand.

Therefore the CMEs were observed much more frequently in cycle 24 compared to cycle 23 . However, their internal structure was also rarefied, so they did not pose a significant threat to our magnetosphere. This effect can simply explain the fact that in cycle 24 more MPEs were caused by CIRs than by CMEs.

Using data included in the CDAW catalog (LASCO and UV images and Dst time profiles) we were able recognize the primary sources of geomagnetic storms associated to the considered MPEs (CME or CIR). Our study shown that MPEs are mostly generated during moderate (associated with CIRs) and intense (associated with CMEs) geomagnetic storms. It turned out that most MPEs were associated with CIRs generated by equatorial solar coronal holes (202 cases). Only 158 MPEs were related to very energetic CMEs.

We demonstrated that the CMEs associated with the observed MPEs were very energetic. They were very wide (halo and partial halo events) with the average speed above $1100 \mathrm{~km} \mathrm{~s}^{-1}$.

CMEs and CIRs generally appear in different phases of solar cycles. MPEs are strongly related to both of these phenomena, therefore they cannot be associated with any phase of a solar cycle or with any indicator characterizing a 11-year sun cycle.

It is also worth noting that the presented results are only related to observations at medium geomagnetic latitudes. They are a very good basis for further, more detailed research on the relationship between MPEs and energetic solar phenomena.

Acknowledgements. The authors were supported by $\mathrm{NCN}$ through the grant UMO-2017/25/B/ST9/00536, and thank Jerzy Kubisz and Adam Michalec from the Astronomical Observatory of the Jagiellonian University for providing and archiving data from the ELF Hylaty station. This work was also supported by a NASA LWS project led by Dr. N. Gopalswamy. We acknowledge use of the SOHO/LASCO CME catalog; this CME catalog is generated and maintained at the CDAW Data Center by NASA and the Catholic University of America in cooperation with the Naval Research Laboratory. We also acknowledge use UV images from Solar Dynamics Observatory (NASA) and Dst data from World Data Center for Geomagnetism, Kyoto. The editor thanks Nadezda Yagova and an anonymous reviewer for their assistance in evaluating this paper.

\section{References}

Akiyama S, Gopalswamy N, Yashiro S, Makela P. 2013. A study of coronal holes observed by SOHO/EIT and the Nobeyama Radioheliograph. Publ Astron Soc Japan 65(S15): https://doi. org/10.1093/pasj/65.sp1.S15.

Anderson B, Erlandson R, Zanetti L. 1992. A statistical study of Pc 1-2 magnetic pulsations in the equatorial magnetosphere 1. Equatorial occurrence distributions. J Geophys Res 97(A3): 3075-3088. https://doi.org/10.1029/91JA02706.

Anderson B, Erlandson R, Zanetti L. 1992b. A statistical study of Pc 1-2 Magnetic Pulsations in the Equatorial Magnetosphere 2. Waves properties. J Geophys Res 97(A3): 3089-3101. https://doi. org/10.1029/91JA02697.

Balmaceda LA, Vourlidas A, Stenborg G, Lago AD. 2018. How reliable are the properties of coronal mass ejections measured from a single viewpoint? Astrophys $J \mathbf{8 6 3}(1)$ : $57,1-16$. https://doi.org/ 10.3847/1538-4357/aacff8.

Benioff H. 1960. Observations of geomagnetic fluctuations in the period range 0.3 to 120 seconds. J Geophys Res 65(5): 1413-1422. https://doi.org/10.1029/JZ065i005p01413.

Bortnik J, Cutler JW, Dunson C, Bleier TE, McPherron RL. 2008. Characteristics of low-latitude Pc1 pulsations during geomagnetic storms. J Geophys Res 113(A04201): 1-9. https://doi.org/10.1029/ 2007JA012867.

Burton RK, McPherron RL, Russell CT. 1975. An empirical relationship between interplanetary conditions and Dst. J Geophys Res 80(31): 4204-4214. https://doi.org/10.1029/JA080i031p04204.

Campbell WH, Stiltner EC. 1965. Some characteristics of geomagnetic pulsations at frequencies near $1 \mathrm{c} / \mathrm{s}$. Radio Sci 69D: 1117-1132.

Cane HV, Richardson IG, Cyr OCS. 2000. Coronal mass ejections, interplanetary ejecta and geomagnetic storms. Geophys Res Lett 27 (21): 3591-3594. https://doi.org/10.1029/2000GL000111.

Carrington R. 1859. Description of a singular appearance seen in the Sun on September 1, 1859. Mon Not Roy Astron Soc 20(1): 13-15. https://doi.org/10.1093/mnras/20.1.13.

Cramer WD, Turner NE, Fok MC, Buzulukova NY. 2013. Effects of different geomagnetic storm drivers on the ring current: CRCM results. J Geophys Res 118: 1062-1073. https://doi.org/10.1002/ jgra.50138.

Cranmer SR. 2002. Coronal holes and the high-speed solar wind. Space Sci Rev 101(3): 229-294. https://doi.org/10.1023/A:1020840004535.

Cranmer SR. 2009. Coronal Holes. Liv Rev Sol Phys 6: https://doi. org/10.12942/lrsp-2009-3.

Cranmer SR, Gibson SE, Riley P. 2017. Origins of the Ambient Solar Wind: Implications for Space Weather. Space Sci Rev 212 (3-4): 1345-1384. https://doi.org/10.1007/s11214-017-0416-y.

Echer E, Tsurutani BT, Gonzalez WD. 2013. Interplanetary origins of moderate $(-100 \mathrm{nT}<\mathrm{Dst}-50 \mathrm{nT})$ geomagnetic storms during solar cycle 23 (1996-2008). J Geophys Res 118: 385-392. https://doi.org/10.1029/2012JA018086.

Engebretson MJ, Lessard MR, Bortnik J, Green JC, Horne RB, et al. 2008. Pc1-Pc2 waves and energetic particle precipitation during and after magnetic storms: Superposed epoch analysis and case studies. J Geophys Res 113(A01211): https://doi.org/10.1029/ 2007JA012362.

Erlandson R, Anderson B. 1996. Pc 1 waves in the ionosphere: A statistical study. J Geophys Res 101(A4): 7843-7857. https://doi. org/10.1029/96JA00082.

Erlandson R, Ukhorskiy A. 2001. Observations of electromagnetic ion cyclotron waves during geomagnetic storms: Wave occurrence and pitch angle scattering. J Geophys Res 106(A3): 3883-3895. https://doi.org/10.1029/2000JA000083. 
Eyles CJ, Harrison RA, Davis CJ, Waltham NR, Shaughnessy BM, et al. 2009. The heliospheric imagers onboard the STEREO mission. Sol Phys 254(2): 387-445. https://doi.org/10.1007/s11207-008-9299-0.

Farrugia CJ, Freeman MP, Burlaga LF, Lepping RP, Takahashi K. 1993. The Earth's magnetosphere under continued forcing: Substorm activity during the passage of an interplanetary magnetic cloud. J Geophys Res 98(A5): 7657-7671. https://doi.org/10.1029/ $92 \mathrm{ja} 02351$.

Fraser-Smith AC. 1970. Some statistics on Pcl geomagnetic micropulsation occurrence at middle latitudes: Inverse relation with sunspot cycle and semiannual period. J Geophys Res 75(25): 4735-4745. https://doi.org/10.1029/JA075i025p04735.

Gonzalez WD, Tsurutani BT, de Gonzalez ALC. 1999. Interplanetary origin of geomagnetic storms. Space Sci Rev 88: 529-562. https://doi.org/10.1023/A:1005160129098.

Gopalswamy N. 2002. Space weather study using combined coronagraphic and in situ observations. COSPAR Colloquia Ser 12: 39-47. https://doi.org/10.1016/S0964-2749(02)80204-9.

Gopalswamy N. 2009. Halo coronal mass ejections and geomagnetic storms. Earth Planets Space 61: 595-597. https://doi.org/10.1186/ BF03352930.

Gopalswamy N, Akiyama S, Yashiro S, Xie H, Mäkelä P, Michalek G. 2014a. Anomalous expansion of coronal mass ejections during solar cycle 24 and its space weather implications. Geophys Res Lett 41(8): 2673-2680. https://doi.org/10.1002/2014GL059858.

Gopalswamy N, Lara A, Kaiser ML, Bougeret JL. 2001a. Near-Sun and nears-Earth manifestations of solar eruptions. J Geophys Res A11: 25261-25277. https://doi.org/10.1029/2000JA004025.

Gopalswamy N, Xie H, Akiyama S, Mäkelä P, Yashiro S, Michalek G. 2015. The peculiar behavior of halo coronal mass ejections in solar cycle 24. Astrophys J Lett 804(1) (L23): 1-6. https://doi.org/ 10.1088/2041-8205/804/1/L23.

Gopalswamy N, Xie H, Akiyama S, Mäkelä PA, Yashiro S. 2014b. Major solar eruptions and high-energy particle events during solar cycle 24. Earth Planets Space 66(104): 1-15. https://doi.org/ 10.1186/1880-5981-66-104.

Gopalswamy N, Yashiro S, Akiyama S. 2016. Unusual polar conditions in solar cycle 24 and their implications for cycle 25. Astrophys J 823(1)(L15): 1-6. https://doi.org/10.3847/2041-8205/ 823/1/L15.

Gopalswamy N, Yashiro S, Kaiser ML, Howard RA, Bougeret J-L. 2001b. Radio signatures of coronal mass ejection interaction: Coronal mass ejection cannibalism? Astrophys J 548: L91-L94. https://doi.org/10.1086/318939.

Gopalswamy N, Yashiro S, Michalek G, Stenborg G, Vourlidas A, Freeland S, Howard R. 2009. The SOHO/LASCO CME Catalog. Earth Moon, Planets 104: 295-313. https://doi.org/10.1007/ s11038-008-9282-7.

Gopalswamy N, Yashiro S, Michalek G, Xie H, Lepping RP, Howard RA. 2005. Solar source of the largest geomagnetic storm of cycle 23. Geophys Res Lett 32(L12S09): 1-5. https://doi.org/ 10.1029/2004GL021639.

Guarnieri FL, Tsurutani BT, Gonzalez WD, Gonzalez ALC, Grande M, Soraas F, Echer E. 2006. ICME and CIR storms with particular emphases on HILDCAA eventsILWS Workshop, February 19-20, GOA.

Guglielmi A, Kangas J. 2007. Pc1 waves in the system of solarterrestrial relations: New reflections. J Atmos Sol Terr Phys 69(14): 1635-1643. https://doi.org/10.1016/j.jastp.2007.01.015.

Guglielmi A, Potapov A, Matveyeva E, Polyushkina T, Kangas J. 2006. Temporal and spatial characteristics of Pc1 geomagnetic pulsations. Adv Space Res 38(8): 1572-1575. https://doi.org/ 10.1016/j.asr.2005.05.027.
Heacock RR, Kivinen M. 1972. Relation of Pc1 micropulsations to the ring current and geomagnetic storms. J Geophys Res 77: 6746-6760.

Jacobs JA, Lokken JE, Wright CS. 1963. Notation and classification of geomagnetic micropulsations. J Geophys Res 68(14): 43734374. https://doi.org/10.1029/JZ068i014p04373.

Kanekal SG. 2006. A review of recent observations of relativistic electron energization in the Earth's outer Van Allen radiation belt. ILWS Workshop-The Solar Influence on the Heliosphere and Earth's Environment, GOA, February 19-24, pp. 1-6.

Kawamura M, Kuwashima M, Toya T, Fukunishi H. 1983. Comparative study of magnetic Pc1 pulsations observed at low and high latitudes: Long-term variation of occurrence frequency of the pulsations. National Institute Polar Research Memoirs 26: $1-12$.

Kerttula R, Mursula K, Pikkarainen T, Kangas J. 2001. Effect of magnetic storm intensity on Pc1 activity at high and mid-latitudes. J Atmos Sol Terr Phys 63(5): 503-511. https://doi.org/10.1016/ S1364-6826(00)00172-3.

Kilpua EKJ, Balogh A, von Steiger R, Liu YD. 2017. Geoeffective properties of solar transients and stream interaction regions. Space Sci Rev 212(3-4): 1271-1314. https://doi.org/10.1007/s11214017-0411-3.

Kim R, Cho K, Moon Y, Kim Y, Yi Y, Dryer M, Bong S, Park Y. 2005. Forecast evaluation of the coronal mass ejection (CME) geoeffectiveness using halo CMEs from 1997 to 2003. J Geophys Res 110(A11104): 1-8. https://doi.org/10.1029/ 2005JA011218.

Kitamura N, Kitahara M, Shoji M, Miyoshi Y, Hasegawa H, et al. 2018. Direct measurements of two-way wave-particle energy transfer in a collisionless space plasma. Science 361(6406): 10001003. https://doi.org/10.1126/science.aap8730.

Kleimenova NG, Kozyreva OV, Breus TK, Rapoport SI. 2007. Pc1 geomagnetic pulsations as a potential hazard of the myocardial infarction. J Atmos Sol Terr Phys 69(14): 1759-1764. https://doi. org/10.1016/j.jastp.2006.10.018.

Klein K, Dalla S. 2017. Acceleration and propagation of solar energetic particles. Space Sci Rev 212(3-4): 1107-1136. https://doi.org/10.1007/s11214-017-0382-4.

Knipp DJ, Fraser BJ, Shea MA, Smart DF. 2018. On the little-known consequences of the 4 August 1972 ultra-fast coronal mass ejecta: facts, commentary and call to action. Space Weather 16(11): 1635-1643. https://doi.org/10.1029/2018SW002024.

Kulak A, Kubisz J, Klucjasz S, Michalec A, Mlynarczyk J, Nieckarz Z, Ostrowski M, Zieba S. 2014. Extremely low frequency electromagnetic field measurements at the Hylaty station and methodology of signal analysis. Radio Sci 49: 361-370. https://doi.org/10.1002/2014RS005400.

Lugaz N, Temmer M, Wang Y, Farrugia CJ. 2017. The interaction of successive coronal mass ejections: A review. Solar Phys 292(64): 1-37. https://doi.org/10.1007/s11207-017-1091-6.

Luhmann JG, Petrie GJD, Riley P. 2013. Solar origins of solar wind properties during the cycle 23 solar minimum and rising phase of cycle 24. J Adv Res 4(3): 221-228. https://doi.org/10.1016/j.jare. 2012.08.008.

Manoharan PK, Agalya G. 2011. High energy solar particle events and their associated coronal mass ejections. Adv Geosci 27: 165-179. https://doi.org/10.1142/9789814355414_0015.

Manoharan PK, Gopalswamy N, Yashiro S, Lara A, Michalek G, Howard RA. 2004. Influence of coronal massejection interaction on propagation of interplanetary shocks. $J$ Geophys Res 109(A06109): 1-9. https://doi.org/10.1029/2003JA010300. 
Märcz F, Verö J. 2002. Pearl-type micropulsations at mid-latitude; their relation to whistlers, solar and geomagnetic activity as well as ionospheric absorption. J Atmos Sol Terr Phys 64(3): 377-387. https://doi.org/10.1016/S1364-6826(01)00102-X.

Mergu RR, Dixit SK. 2011. Multi-Resolution Speech Spectrogram. Int J Comput Appl 15(4): 28-32. https://doi.org/10.5120/19372587.

Michalek G, Gopalswamy N, Yashiro S. 2019. On the coronal mass ejection detection rate during Solar Cycles 23 and 24. Astrophys $J$ 880:51(1): 1-16. https://doi.org/10.3847/1538-4357/ab26a7.

Moon Y-J, Cho K-S, Dryer M, Kim Y-H, Chan Bong S, Chae J, Park YD. 2005. New geoeffective parameters of very fast halo coronal mass ejections. Astrophys J 624(1): 414-419. https://doi.org/ 10.1086/428880.

Mursula K, Anderson B, Erlandson R, Pikkarainen T. 1996. Solar cycle change of $\mathrm{Pcl}$ waves observed by an equatorial satellite and on the ground. Adv Space Res 17(10): 51-55. https://doi.org/ 10.1016/0273-1177(95)00694-A.

Mursula K, Kangas J, Pikkarainen T, Kivinen M. 1991. Pc1 micropulsations at a high-latitude station: A study over nearly four solar cycles. J Geophys Res 96(A10): 17651-17661. https://doi. org/10.1029/91JA01374.

Navia CE, de Oliveira MN, Augusto CRA. 2018. The highest geomagnetic storms of the solar cycle observed at ground level. In: Extreme Weather. Sallis PJ, (Ed.) IntechOpen, pp. 31-47.

Nieckarz Z. 2016. Imprints of Natural Phenomena and Human Activity Observed During 10 Years of ELF Magnetic Measurements at the Hylaty Geophysical Station in Poland. Acta Geophys 64(6): 2591-2608. https://doi.org/10.1515/acgeo-2016-0101.

Nomura R, Shiokawa K, Pilipenko V, Shevtsov B. 2011. Frequencydependent polarization characteristics of $\mathrm{Pc} 1$ geomagnetic pulsations observed by multipoint ground stations at low latitudes. J Geophys Res 116(A01204): https://doi.org/10.1029/2010JA015684.

Norton H, Newmark JS, Feldman U. 1999. SOHO EIT observations of coronal holes. In: Proceedings of the Conference held 22-25 June 1999 in CAP 15, 1-13 Quai de Grenelle, 75015 Paris, France, pp. 446-509.

Park J, Luhr H, Rauberg J. 2013. Global characteristics of Pc1 magnetic pulsations during solar cycle 23 deduced from CHAMP data. Ann Geophys 31: 1507-1520. https://doi.org/10.5194/angeo31-1507-2013.

Paulson KW, Smith CW, Lessard MR, Torbert RB, Kletzing CA, Wygant JR. 2017. In situ statistical observations of Pc1 pearl pulsations and unstructured EMIC waves by the Van Allen Probes. J Geophys Res 122: 105-119. https://doi.org/10.1002/ 2016JA023160.

Pesnell WD. 2016. Predictions of solar cycle 24: How arewe doing? Space Weather 14(1): 10-21. https://doi.org/10.1002/2015SW001304.

Petrie GJD. 2013. Solar magnetic activity cycles, coronal potential field models and eruption rates. Astrophys $J$ 768(162): 1-18. https://doi.org/10.1088/0004-637X/768/2/162.

Petrie GJD. 2015a. On the enhanced coronal mass ejection detection rate since the Solar Cycle 23 Polar field reversal. Astrophys $J \mathbf{8 1 2}$ (74): 1-14. https://doi.org/10.1088/0004-637X/812/1/74.

Petrie GJD. 2015b. Solar magnetism in the polar regions. Liv Rev Solar Phys 12(5): 84-89. https://doi.org/10.1007//rsp-2015-5.

Plotnikov I, Rouillard AP, Davies JA, Bothmer V, Eastwood JP, et al. 2016. Long-Term Tracking of Corotating Density Structures Using Heliospheric Imaging. Solar Phys 291: 1853-1875. https://doi.org/10.1007/s11207-016-0935-9.

Posch JL, Engebretson MJ, Murphy MT, Denton MH, Lessard MR, Horne RB. 2010. Probing the relationship between electromagnetic ion cyclotron waves and plasmaspheric plumes near geosynchronous orbit. J Geophys Res 115(A11205): 1-18. https://doi.org/10.1029/2010JA015446.

Richardson IG, Cane HV. 2012a. Near-earth solar wind flows and related geomagnetic activity during more than four solar cycles (1963-2011). J Space Weather Space Clim 2(A02): 1-10. https://doi.org/10.1051/swsc/2012003.

Richardson IG, Cane HV. 2012b. Solar wind drivers of geomagnetic storms duringmore than four solar cycles. J Space Weather Space Clim 2(A01): 1-9. https://doi.org/10.1051/swsc/2012001.

Richardson IG, Cliver EW, Cane HV. 2001. Sources of geomagnetic storms for solar minimum and maximum conditions during 19722000. Geophys Res Lett 28(13): 2569-2572. https://doi.org/ 10.1029/2001GL013052.

Richardson IG, Webb DF, Zhang J, Berdichevsky D, Biesecker DA, et al. 2006. Major geomagnetic storms (Dst $\leq-100 \mathrm{nT}$ ) generated by corotating interaction regions. J Geophys Res 111(A7): 1-17. https://doi.org/10.1029/2005ja011476.

Schrijver CJ. 2015. Socio-Economic Hazards and Impacts of Space Weather: The Important Range Between Mild and Extreme. Space. Weather 13(9): 524-528. https://doi.org/10.1002/2015sw001252.

Scolini K, Chane E, Temmer M, Kilpua EKJ, Dissauer K, Veronig AM, Dumbovic EPJPM, Guo J, Rodriguez L, Poedts S. 2020. CME-CME Interactions as Sources of CME Geoeffectiveness: The Formation of the Complex Ejecta and Intense Geomagnetic Storm in 2017 Early September. Astrophys J Suppl Ser 247(21): 1-27. https://doi.org/10.3847/1538-4365/ab6216.

Shanmugaraju A, Ibrahim MS, Moon Y-J, Rahman AM, Umapathy S. 2015. Empirical Relationship Between CME Parameters and Geo-effectiveness of Halo CMEs in the Rising Phase of Solar Cycle 24 (2011-2013). Solar Physics 290: 14171427. https://doi.org/10.1007/s11207-015-0671-6.

Shen C, Wang Y, Pan Z, Zhang M, Ye P, Wang S. 2013. Full halo coronal mass ejections: Do we need to correct the projection effect in terms of velocity? J Geophys Res 118(11): 6858-6865. https://doi.org/10.1002/2013ja018872.

Stewart B. 1861. On the great magnetic disturbance of August 28 to September 7, 1859, as recorded by photography at the kew observatory. Proc Roy Soc London 11: 407-410.

Subrahmanyam S, Narayan PVS, Srinivasan TM. 1985. Effect of magnetic micropulsations.on the biological systems - a bioenvironmental study. Int J Biometeor 29(3): 293-305.

Temmer M, Preiss S, Veronig AM. 2009. CME projection effects studied with STEREO/COR and SOHO/LASCO. Solar Phys 256 (1-2): 183-199. https://doi.org/10.1007/s11207-009-9336-7.

Verbanac G, Vršnak B, Veronig A, Temmer M. 2011. Equatorial coronal holes, solar wind high-speed streams, and their geoeffectiveness. Astron Astrophys 526(A20): 1-13. https://doi.org/ 10.1051/0004-6361/201014617.

Vršnak B, Ruzdjak D, Sudar D, Gopalswamy N. 2004a. Kinematics of coronal mass ejections between 2 and 30 solar radii - What can be learned about forces governing the eruption? Astron Astrophys 423: 717-728. https://doi.org/10.1051/0004-6361:20047169.

Vršnak B, D, AL. 2004b. Coronal mass ejection of 15 May 2001: II. Coupling of the CME acceleration and the flare energy release. Solar Phys 225: 355-378. https://doi.org/10.1007/s11207-004-4995-x.

Wang Y-M, Colaninno R. 2014. Is solar cycle 24 producing more coronal mass ejections than cycle 23? Astrophys J Lett 784(L27): 1-8. https://doi.org/10.1088/2041-8205/784/2/L27.

Watari S. 2017. Geomagnetic storms of cycle 24 and their solar sources. Watari Earth Planets Space 69(70): 1-8. https://doi.org/ 10.1186/s40623-017-0653-z. 
Webb DF, Howard RA, Cyr OCS, Vourlidas A. 2017. Is there a CME rate floor? CME and magnetic flux values for the last four solar cycle minima. Astrophys J 851(142): 1-10. https://doi.org/ 10.3847/1538-4357/aa9b81.

Wentworth RC. 1964. Enhancement of hydromagnetic emissions after geomagnetic storms. J Geophys Res 69: 2291-2298.

Yashiro S, Gopalswamy N, Michałek G, Cyr O, Plunkett S, Rich NB, Howard RA. 2004. A catalog of white light coronal mass ejections observed by the SOHO spacecraft. J Geophys Res 109(A07105): 1-11. https://doi.org/10.1029/2003JA010282.

Zhang J, Dere KP, Howard RA, Bothmer V. 2003. Identification of Solar Sources of Major Geomagnetic Storms between 1996 and
2000. Astrophys J 582(1): 520-533. https://doi.org/10.1086/ 344611.

Zhang J, Dere KP, Howard RA, Kundu MR, White SM. 2001. On the temporal relationship between coronal mass ejections and flares. Astrophys J 559(1): 452-462. https://doi.org/10.1086/322405.

Zhang J, Richardson IG, Webb DF, Gopalswamy N, Huttunen E, et al. 2007. Solar and interplanetary sources of major geomagnetic storms (Dst $=-100 \mathrm{nT}$ ) during 1996-2005. J Geophys Res 112(A10102): https://doi.org/10.1029/2007JA012321.

Zieba S, Nieckarz Z. 2014. Sunspot time series: passive and active intervals. Solar Phys 289(7): 2705-2726. https://doi.org/10.1007/ s11207-014-0498-6.

Cite this article as: Nieckarz Z \& Michałek G 2020. Long-term observation of magnetic pulsations through the ELF Hylaty station located in the Bieszczady Mountains (south-eastern Poland). J. Space Weather Space Clim. 10, 59. https://doi.org/10.1051/swsc/2020063. 